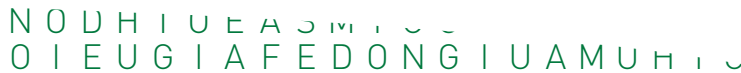

$H R X S A D B H M G R$ I BDPBDLRBEFBAr.

$U D M P X P|Z R W Q S C G Z N J| M N S T R V N P \mid \angle N$

A A T S I M U L A T I O N A R N Z F G D L I ER N G M J b .

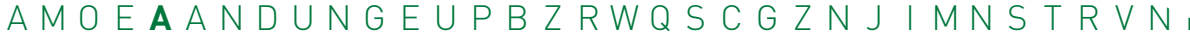

EK J I LKO I J GRDCKI O PMNESWLNCAWZ YKFEQLO,

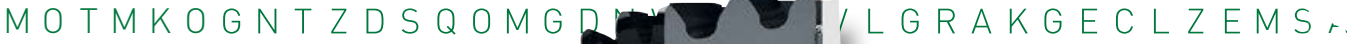

TNUGFNRLUJGD|NGD—: TFNAXJRCNIFZKM,

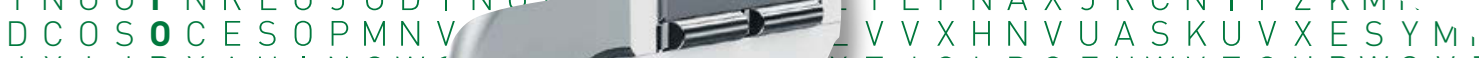

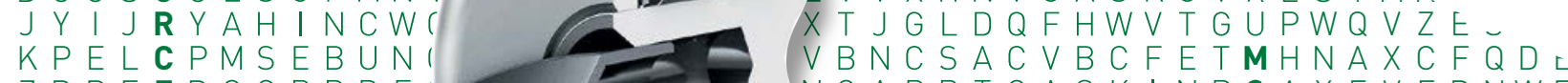
ZBPEEBQOPBDEI NCAPRTCASKINROAXEVEDNWE TNEHBNZWEDCY I GTGSGEBCRZYDINDERSTJK $C R O E T R W P O P$
$B E F S H E C E F$ $S O B P \mid O S G$ F E I WR E Q R CWD A Y T T R $P J M T \mid J H$ C G T B D G L $J T Z$ I E T O VWMWR W U A KDS J KF $L S J A D S$ EK J B C K $M O T P Q O$ $T N \cup A \mid N$, DCONVCE $J \quad Y \quad I \quad Z \quad Q \quad Y \quad A$. $N J K R N J R A$ $A A O L U N D$

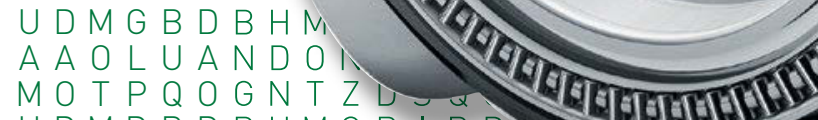

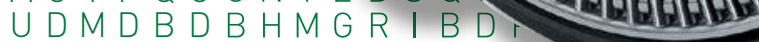
$F E|H R E Q R| U Z T R E W Q D$ CLETUOADGJLYCBMWRZIPSFHK R KTVNZLMOIJEUHBZOQA $P J M T I J H L M O K N I J U H B Z G V T F C R D X L L K H E S Y S C B F G M H T I L Q T F$ C GTJDGLETUOADGJLYCBMWRZIPSFPONCALVIKNDVSGWJGWR J T Z UETOIZRWQETUOMBCYNVXADGJCVBNMIQWURTZBCSDQN 


\section{Turning New Directions: Surprising Potential in Planetary Transmissions}

\section{Part 1: Planetary gear set}

Rainer Schuebel

Martin Gegner

Frank Beeck

D v

$J \mathrm{ZMH}_{\llcorner}$

$A G Q S W \mid\llcorner\cdots$

F I MBCHSEH

I C E C B S T P O I O D C V ז
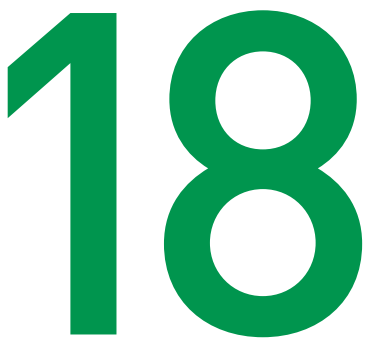

$D G \vee T Q U J X R E L K J H G F D S A|V| \cdots$

YLMRTXAGYWPHCEQA YWS XE E C K.

CXVNHOUB I JBZGVTFCRDXESNWASKL

$Z V T F L U J A D G Y C B M W R Z$ I P S F H K T VNZLMU,

$X D B P O R U T E T M B C Y N V X A D G J L K H E S Y S C B M B \iota$

DCSKUPOWRWZTWHNEDKUNWPONCALVIKZTWHIV

EHKLPFLKJKO I UZTREWQYXCVBNM I QWUO I UZTR

WDXAYHASGSVNPIZRWQSCGZNJ|MNSTRVNPIZRWQ_

I L Z UKOGIKCKPMNESWLNCXWZYKFEDIOPPMNESWLNCX

$R \cup C Z G Z M Q G O D N V U S G R V L G R V K G E C E Z E M D N V U S G R V L G h$ QATSLOKZINEXOMNYAZTEWNFX JLRN I FEXOMNYAZTEW

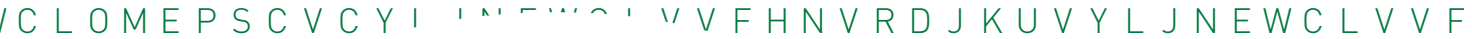
FAMUAN J Y $\cap \cdots$

KMN S R D O

LI E P N N

B S A T B

$P \mid E P$ P

$R \cup C Z$

B S A

$D G \vee$

$Y\llcorner\wedge$

C X

Z V

$X \mathrm{D}$ $S Q F H B Q F G O B R E L N F X T J C$ I ZPMFDR O I D F G KLDF I $A \cup K Z Q H|O G D N O| E R N L$

.1 I O G D O I EK.

D D L R B E F A F V N K

. UAH I OGDNO| ER N G M

OQODNVUSGRVLGRVKG $P D B D D L R B E F B A F \vee N K F N$ r

, OTRELKJHGFDSAMMBVCX $X A Z Y W P H C E Q A Y W S X E E C R F I$ $O U B$ I JBZGVTFCRDXESNWA S U JRDGYCBMWRZIPSFHKTVN $R \cup T E T M B C Y N \vee X A D G J L K H E S$ P OWRWZTWHNEDKUNWPONCA $F L K J K O \mid U Z T R E W Q Y X C \vee B N M$ HASESVNPIZRWQSCGZNJIM GIKCKPMNESWLNCXWZYKFF I A S U S V N P I Z R Q S C G Z N J | I KCKPMNESWLNCXWZYKF 'OXODNVUSGRVLGRVKG

1 I NEXOMNYAZTEWNF

' C Y L J EWCLVVFH' $\cap N \vee \cup S G R \vee L G F$
REWSPDLRB EFBAFVNK

L K Z Q H I O GDNO।ERNGN Z E M D N U S G R V L R R K NSPDLRBEFBAFVNKF $M O L K J H G F D S A M M B V$ $B Z P H C E Q A Y W S X E E C$ ¿ VBZGVTFCRDXESN 10 Y C B MWR Z I P S F H K B M B C Y N V X A D J L K K Z TWHNEDKUNWPr 


\section{Introduction}

The automotive industry and suppliers have implemented numerous innovations with the objective of reducing the $\mathrm{CO}_{2}$ emissions of individual transport. Examples are general lightweight designs and optimizations to the exhaust gas system as well as numerous detailed solutions for engine technology. For many years, transmission technology has also been contributing to the continuous reduction of fuel consumption and emissions. This has usually been accompanied by an increase in the number of gears. This increased number of gears and the smaller transmission ratio spread result in smooth, more comfortable and hardly perceivable gearshift operations. At the same time, each additional gear has enabled reductions in fuel consumption and emissions by several percentage points by approximating the optimal tractive force hyperbola (Figure 1).

With the increased number of gears, the number of planetary gear sets in automatic transmissions also tended to be increased. This trend was not linear in relation to the number of gears due to the

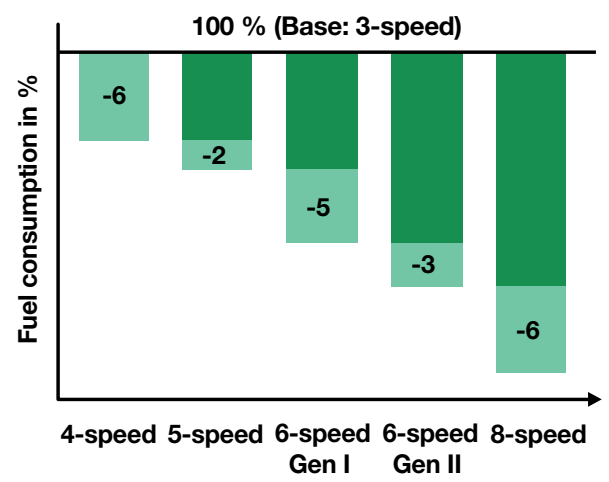

Figure 1 Reductions in fuel consumption based on transmission development intelligent control of the flows of force. The design envelope of the transmission, however, remained the same. The individual transmission components therefore had to become smaller and more compact. This requirement often created special challenges for the design and dimensioning of components. At the same time, the requirements for the materials and manufacturing technologies used have increased.

Schaeffler has been able to make significant contributions to reducing emissions and fuel consumption by continuously optimizing planet gear bearings and axial needle roller bearings. Recent analyses have shown that even inconspicuous new developments can offer great potential. The most recent example is the new axial needle roller bearing support for planet gears. This development is considered a first in rolling bearing technology and can contribute to reducing $\mathrm{CO}_{2}$ emissions by up to $1 \mathrm{~g} / \mathrm{km}$ with low additional costs.

\section{Trends and challenges}

The further development of transmission technology has increased the subsequent requirements for modern planetary gear sets by more than $50 \%$ during the last few years. This is because fuel consumption can only be reduced by means of smaller jumps in speed, which requires a wider transmission ratio spread and causes additional outlay for the design of the transmission, for example due to an additional planetary gear set required. The center distances in the planetary gear set are also becoming larger in order to achieve the required forces and moments through the ratios. The center distance 


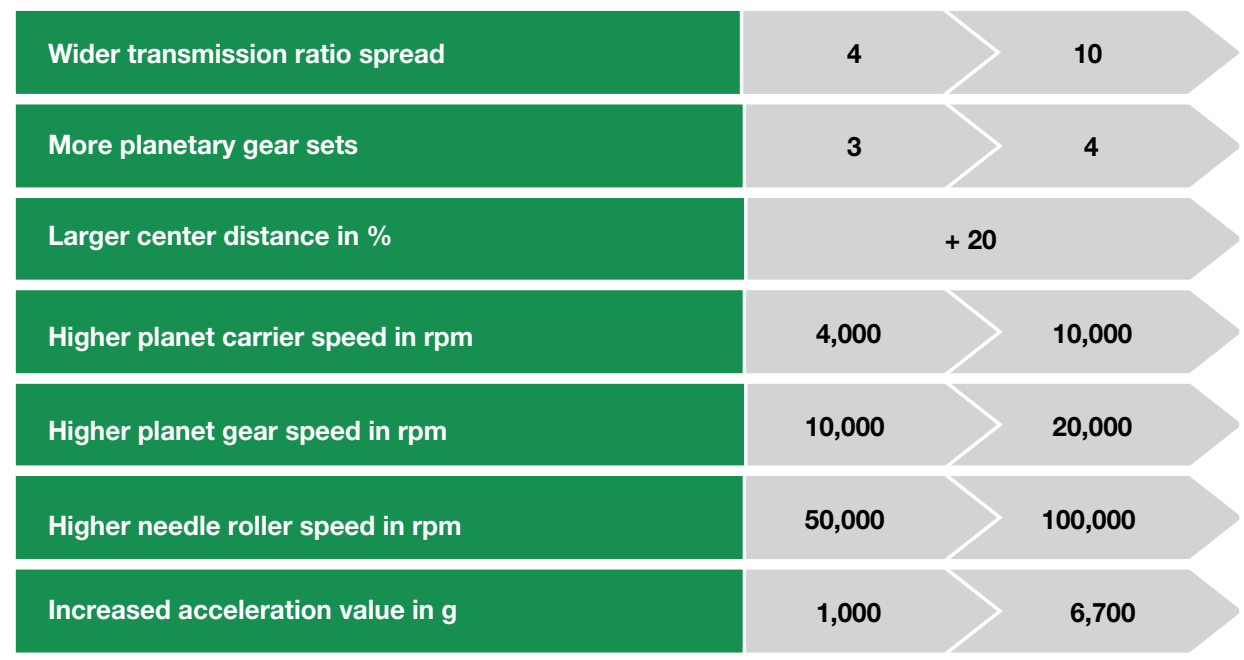

Figure 2 Challenges for the transmission and effects on the components

and the speed of the planet carrier cause high centrifugal force loads at individual operating points of the transmission. Often, the centrifugal forces even increase considerably. This results in higher loads acting on the planet carrier, the planet gear, and the planet gear bearing. The maximum carrier speed and the maximum centrifugal acceleration are now slightly above the currently valid limits. However, these values can be controlled with adequate development outlay.

The number of gears in automatic transmissions can be increased by using additional shift elements or more planet stages. Looking back at the six-speed automatic transmission, the number of gears could be increased further by using a fourth planetary gear set. This additional planet stage has proved ideal with regard to constant or reduced drag losses due to open shift elements. [1]

Certain cases, however, require an automatic transmission with a particularly space-saving design in order to use it in front transverse applications, for example. The most suitable method for saving space is the nesting of planetary gear sets. As a result, the outer nested gear set has a comparably large center distance (Figure 2) [2].

Previous requirements for planetary gear sets have been implemented by using state-of-the-art bearing supports for the planet gears. This means that bearing loads of up to 3,500 times the acceleration due to gravity $\left(g=9.81 \mathrm{~m} / \mathrm{s}^{2}\right)$ can be managed using current manufacturing methods. However, the latest generation of automatic transmissions must meet bearing load specifications of up to $6,700 \mathrm{~g}$, and future transmissions must even be designed for up to $8,000 \mathrm{~g}$. The basis for this is the dependency of the centrifugal acceleration on the speed of the load-bearing component (Figure 3).

The following comparisons can give a better idea of the occurring forces. For example, forces of around $4 \mathrm{~g}$ act on the human body in a carousel. A fighter pilot is subjected to up to $25 \mathrm{~g}$ when the ejection seat is fired. The forces of up to $6,700 \mathrm{~g}$ acting on a planet gear bearing can also be explained using this example: If a nee- 


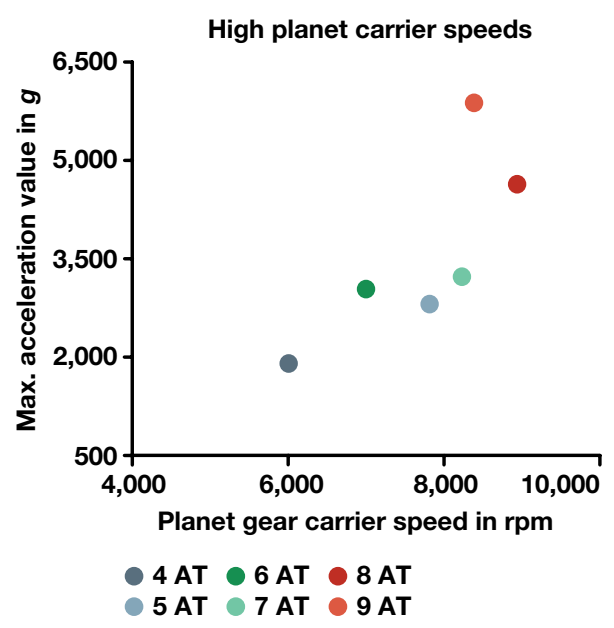

Figure 3 Changes in planet carrier speed

dle roller in a planet gear bearing weighs one gram, an acceleration of $6,700 \mathrm{~g}$ results in a mass of around $6.7 \mathrm{~kg}$. Such values push the limits of design and materials technology and require innovative development solutions.

\section{Power losses}

Even though transmissions generally have relatively high levels of efficiency, losses cannot be completely prevented in individual assemblies. They are mainly caused by the lockup clutch and its actuation system in the converter. The one-way clutch supporting the stator is considered as relatively negligible in this regard. The adjacent oil pump is increasingly actuated by means of a separate electromechanical system. This means that energy must be used only when a specific oil quantity or oil pressure is required. The focus of planetary gear sets is on the influence of centrifugal force, the rolling friction of the gear teeth, the bearing friction, and the axial sliding friction of the planet gears. The main focus of bearings for automatic transmission components is on axial bearings, ball bearings, or radial plain guidance systems. Additional losses are due to the viscosity of the oil, the throttling/pump effects of various rotating components, and churning losses.

\section{Reducing frictional power}

\section{Planet gear bearings in general}

The main purpose of planet gear bearings is to position the planet gears [3]. In addition, they must support forces and moments and ensure rotation of the planet gears with minimal friction. Bearings with needle roller and cage assemblies are mainly used due to the speed and centrifugal force requirements. The cage, which is usually made of steel, guides and positions the rolling elements. The cages are generally manufactured using forming, punching and welding methods in order to ensure efficient production of the high quantities required for automotive applications.

The raceways for the rolling bearing are located directly on the gear set components. The outer raceway is defined by the bore of the planet gear. The stud that is firmly located in the planet carrier provides the inner raceway. Special thrust washers facilitate the axial contact between the planets and the bearing cages. This design represents a cost-effective and highly functional bearing arrangement. 
The bearing itself comprises a specific number of load-bearing rolling elements (needle rollers) and a so-called cage, which guides the needle rollers both axially (cage rib) and in circumferential direction (cage crosspieces). The cage crosspieces have outer and inner retentions that retain the needle rollers radially and prevent them from falling out. The position of these retentions has been optimized so that cage stresses can be kept as low as possible. The pocket corner radii have also been optimized in order to reduce the component stresses.

\section{Cage design}

Another important point in the design of planet gear bearings is the friction behavior. If the diameter, length or number of rolling elements are modified, this can result in significant changes in friction. The following comparison of the calculated friction components of two bearing design alternatives subjected solely to centrifugal force loads demonstrates this as an example. Compared to the standard design with rolling elements with a diameter of $2.5 \mathrm{~mm}$, the alternative has thinner rolling elements with a diameter of $2.0 \mathrm{~mm}$ and therefore a slightly larger inner raceway diameter. With the same static load ratings, the friction of the rolling elements with the smaller diameter is reduced by around $11 \%$.

Cage friction depends on the selected rolling element diameter and therefore has a significant influence on the friction behavior of the bearings. This presents varied challenges for the design of planet gear bearings as it must combine sufficient load ratings and cage strength with minimal friction.

\section{Coating}

Adapted coatings for bearing cages also play an important role in increasing the efficiency of planet gear bearings. The bearing cage is subjected to sliding contact due to the occurring loads. For example, the rolling elements are pressed against the outer retentions of the crosspieces when subjected to centrifugal force, and the outside surface of the cage is pressed into the planet bore.

Simulations have shown that optimizing the contact surfaces of the cage is advantageous as they account for up to $70 \%$ of the friction. The rolling surfaces of the needle rollers and the raceways are less suitable for low-friction coatings as they already meet the highest surface requirements in order to function as rolling raceways.

Coatings based on zinc phosphate (Durotect Z) or manganese phosphate (Durotect $M$ ) improve the sliding and wear behavior of metal contact surfaces that slide against each other and also contribute to corrosion protection. Due to their capability of storing oil, the layers are also used specifically for sliding contact surfaces. The previously used Durotect Z layer has been replaced by the Durotect $M$ layer for planet bearing applications. Practical experience has shown that this layer achieves significant advantages in terms of friction and wear.

If additional increases in efficiency are required, a specially developed coating based on nickel and phosphorus (Durotect NP) is used on the bearing cages to reduce friction. This layer offers very good adhesive wear resistance, excellent dry running characteristics, and temperature resistance in combination with outstanding sliding and anti-adhesive characteristics.

The measured frictional power using Durotect $\mathrm{M}$ is approximately $10 \%$ lower 
compared to the previously used Durotect $Z$ layer. A significant reduction in wear has also been achieved. The Durotect NP coating additionally reduces friction by a further $13 \%$.

\section{Planet gear design}

In order to investigate possible optimizations for planet gear design, Schaeffler modified the parameters of the bearing support of a planet gear while maintaining the same gear teeth. The objective was to achieve the maximum possible planet gear inside diameter, which at the same time requires the minimum possible wall thickness and the maximum possible outer raceway of the bearing. Varying rolling element diameters and the correspondingly required number of rolling elements in the pitch circle diameter were used to determine the radial bearing support geometry with the longest rating life. This specified geometry also determines the raceway inside diameter and the size of the planet gear stud.

The smaller wall thickness reduces the mass of the planet gear and the resulting centrifugal forces.
The investigations led to the following conclusions: The strategy of using a smaller rolling element diameter and subsequently increasing the number of rolling elements results in a bearing support with reduced friction and a higher load carrying capacity. Calculations have shown an increase in rating life by $55 \%$ for the optimized bearing in conjunction with a reduction in the planet gear mass by $30 \%$. This leads to a reduction in friction by $50 \%$ in the radial bearing and a reduction in the centrifugal force by $60 \%$. Figure 4 provides an overview of the successes achieved in development.

\section{Latest findings from axial bearing supports for planet gears}

In a planetary gear set, the annulus, the planet gear and the sun wheel mesh with each other. The planet gear plays a special role as it meshes with both the annulus and the sun wheel. The gear teeth

\begin{tabular}{|l|c|c|}
\hline $\begin{array}{l}\text { Optimizations compared } \\
\text { with the previous product }\end{array}$ & $\begin{array}{c}\text { Friction behavior } \\
\text { compared with the } \\
\text { standard as basis } \\
(100 \%)\end{array}$ & $\begin{array}{c}\text { Responsibility } \\
\text { for development }\end{array}$ \\
\hline $\begin{array}{l}\text { Optimized planet gear bearing } \\
\text { cage design }\end{array}$ & $-11 \%$ & Schaeffler \\
\hline $\begin{array}{l}\text { Optimized planet gear bearing } \\
\text { cage coating }\end{array}$ & $-23 \%$ & \begin{tabular}{c} 
Schaeffler \\
\hline $\begin{array}{l}\text { Weight-optimized } \\
\text { planet gear }\end{array}$
\end{tabular} \\
\hline
\end{tabular}

Figure 4 Overview of improvements 

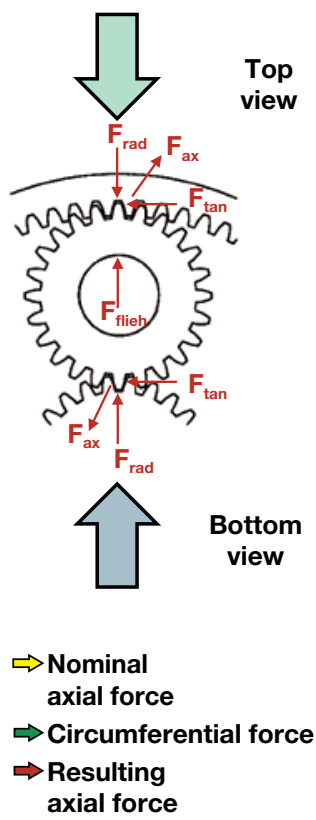
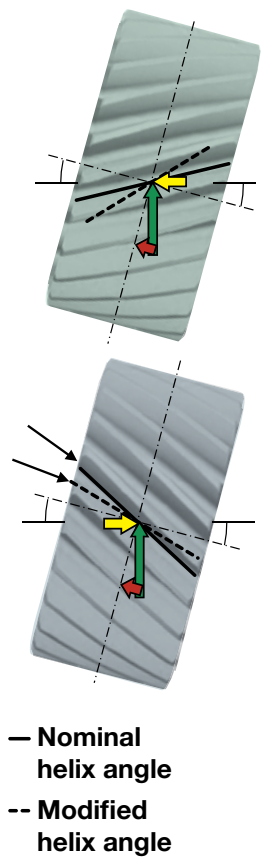

Figure 5 Force conditions on the planet gear

are helical. Meshing forces are generated when the planet gear meshes with the annulus and the sun wheel. They cause a force that acts on the planet gear stud in circumferential direction (Figure 5).

Furthermore, centrifugal forces occur when the planet carrier rotates. The rotation of the planet gear generates frictional forces due to the contact with the planet gear bearing. Forces are also generated by the angular acceleration of the planet gear, and additional frictional forces result from the rolling contact itself. All three forces act against one of the two meshing forces depending on the direction of the power flow. This causes irregularities in the sun/planet and planet/annulus systems, resulting in an axial force that acts on the end faces of the planet gears (red arrows).
As part of the development work in this field, Schaeffler modified the last position without rolling bearing supports in the planetary gear set. This position has a sliding contact surface. It comprises either a nonferrous metal washer or a steel washer, and sometimes also a combination of materials. The non-ferrous metal washer is the friction partner for the planet carrier made of unhardened steel. The steel washer is used as a thrust washer for the planet gear and its bearing.

The plain washers are very small. They can have various characteristics despite their dimensions, for example an inside diameter of $17 \mathrm{~mm}$, an outside diameter of $30 \mathrm{~mm}$, and a thickness of $1 \mathrm{~mm}$. These include:

- special oil feed grooves or oil ways,

- retentions for simplified final assembly,

- anti-rotation locking devices (to prevent abrasive wear), and

- various coatings.

The small design envelope represents a major challenge for the development of an adequate rolling bearing. A reliable standard axial needle roller bearing has a product width of $2.13 \mathrm{~mm}$. The needle roller diameter is $1.5 \mathrm{~mm}$, and the washer thickness is $0.63 \mathrm{~mm}$.

The objective was to develop a new axial needle roller bearing with the same smaller dimensions. Schaeffler has successfully achieved this with its latest axial needle roller bearing with an axial washer. It has an inside diameter of $17 \mathrm{~mm}$, an outside diameter of $29.9 \mathrm{~mm}$, and a thickness of $1.2 \mathrm{~mm}$. Schaeffler was able to reduce the needle roller diameter to $1.0 \mathrm{~mm}$ and the washer thickness to $0.2 \mathrm{~mm}$ (Figure 6).

This design places very high demands on the quality of the material and its surface and heat treatment in order to fulfill the requirements for rolling bearings and withstand the occurring loads. The filmlike washer thickness also represents a special challenge for the production pro- 


\begin{tabular}{|l|c|c|c|}
\hline \multicolumn{1}{|c|}{ Dimensions } & Thrust washer & $\begin{array}{c}\text { New axial needle } \\
\text { roller bearing }\end{array}$ & $\begin{array}{c}\text { Standard axial needle } \\
\text { roller bearing }\end{array}$ \\
\hline Inside diameter & $17 \mathrm{~mm}$ & $17 \mathrm{~mm}$ & $50.8 \mathrm{~mm}$ \\
\hline Outside diameter & $30 \mathrm{~mm}$ & $29.9 \mathrm{~mm}$ & $67.5 \mathrm{~mm}$ \\
\hline Width & $1.0 \mathrm{~mm}$ & $1.2 \mathrm{~mm}$ & $4.0 \mathrm{~mm}$ \\
\hline Needle roller dimensions & - & $1.0 \times 2.25 \mathrm{~mm}$ & $3 \times 4.3 \mathrm{~mm}$ \\
\hline Washer thickness & $1.0 \mathrm{~mm}$ & $0.2 \mathrm{~mm}$ & $1.0 \mathrm{~mm}$ \\
\hline Needle roller speed & - & $500,000 \mathrm{rpm}$ & $120,000 \mathrm{rpm}$ \\
\hline
\end{tabular}

Figure 6 Modifications to the axial planet gear bearing support

cess. The needle roller with a diameter of $1 \mathrm{~mm}$ and a length of $2.25 \mathrm{~mm}$ is the smallest rolling element ever used in transmission applications. The axial needle roller cage must have a very filigree design in order to securely guide and retain the needle rollers.

The behavior of the axial bearing support in the planetary gear set for an entire transmission and its effects on fuel consumption were investigated for four differ- ent planetary gear sets using simulation tools. The simulation was based on the NEDC with a reduced number of load points of 1,400 . The engine data map based on the NEDC and the mass inertia values correspond to those of a premium vehicle. The friction parameters were determined on the basis of test stand runs (Figure 7) [4, 5].

The results in Figure 8 show a comparison of the power loss of the individual plan-

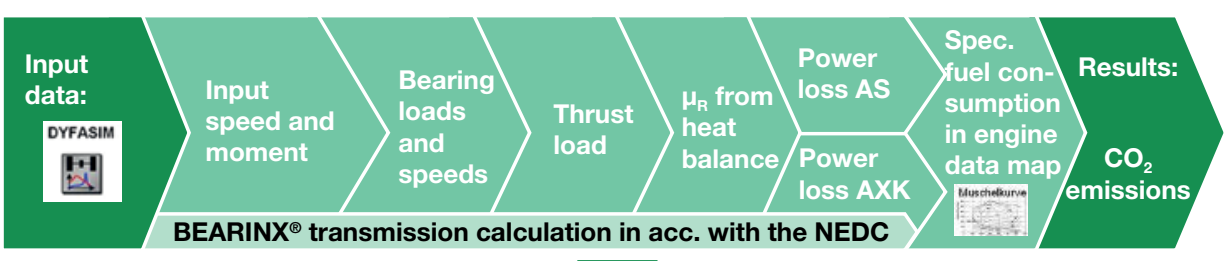

Friction parameters $\mu_{\mathrm{R}}$ from the heat balance

\begin{tabular}{|c|c|c|c|c|c|}
\hline Scenario & Thrust load & Churning & Wear & P_AXK (basis) & $\mu_{\mathrm{R}_{2}}$ AS \\
\hline 1 & $3.5 \%$ & yes & yes & $74.40 \mathrm{~W}$ & 0.018 \\
\hline 2 & $7.0 \%$ & yes & - & $14.88 \mathrm{~W}$ & 0.070 \\
\hline
\end{tabular}

AS: Axial washer AXK: Axial needle roller and cage assembly
P_AXK: Power loss of axial needle roller and cage assembly

Figure 7 Simulation 


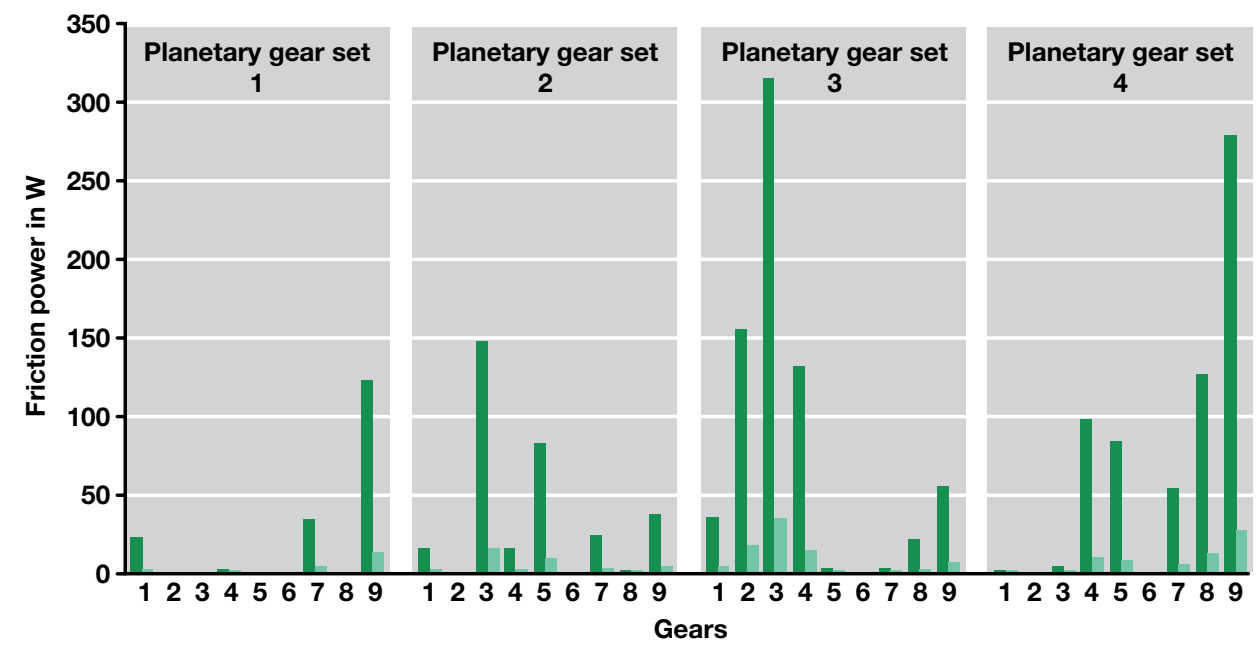

Frictional power with thrust washer

Frictional power with axial needle roller bearing

Figure $8 \quad$ Simulation results

etary gear sets under various load conditions using plain bearings or rolling bearings. The values can be accumulated for the relevant gears only. Under the specified load conditions, the axial bearing achieves relatively high values in two planetary gear sets, which represent a highly effective reduction in friction if a suitable rolling bearing support is used.

In the third gear, for example, the maximum frictional power is $470 \mathrm{~W}$ if a thrust washer is used, but only $50 \mathrm{~W}$ if an axial needle roller bearing support is used. For the entire transmission with four planetary gear sets, this means a reduction in frictional power by $420 \mathrm{~W}$ or $90 \%$ in the third gear. Based on the simulation, a reduction in fuel consumption by around $0.5 \%$ in the NEDC can be expected if the plain washers are replaced with axial needle roller bearings.

Comparative tests with axial bearings and plain washers on an axial bearing highspeed test stand also confirmed reduced frictional torque and additional temperature differences. To determine the speed limits of the axial bearing depending on the axial load, the oil temperature and the oil flow rate, the axial load is introduced into the test stand using a hydraulic system. The hydrostatic system enables measurements of the frictional torques at high speeds.

Speeds of 6,000 rpm and 20,000 rpm were specified as test conditions. The axial load was $500 \mathrm{~N}$. A reduction in frictional torque from $0.23 \mathrm{Nm}$ to $0.024 \mathrm{Nm}$ was achieved at a speed of $6,000 \mathrm{rpm}$. At a speed of 20,000 rpm, the frictional torque was reduced from $0.13 \mathrm{Nm}$ to $0.03 \mathrm{Nm}$. This means that the frictional torque can be reduced by around $90 \%$ with the new axial needle roller bearing. At the same time, the temperature on the bearing position decreases by 5 to $10^{\circ} \mathrm{C}$ (Figure 9).

As an alternative to complex and costly tests with planetary gear sets in entire transmissions, Schaeffler has a component test stand that provides the option of investigating the function and operating life of entire planetary gear sets subjected to centrifugal force and specified loads. The moment is variably introduced using two coupled plan- 
etary gear sets. Additional influencing parameters of the test setup are the supplied quantity of oil and its temperature for lubricating and cooling the gear set. The measured bearing temperature has proven to be a reliable inspection criterion for monitoring. Temperature sensors measure the temperature directly in the gear set on each planet gear bearing support. This enables conclusions to be drawn about the functional capability and the behavior of the system during the test. If a sudden increase in temperature is measured, this is a reliable indication of damage to the planet gear bearing support. In most cases, this means that the bearing cage is defective.

This test stand can also be used for comparative tests with planetary gear sets using axial bearings and planetary gear sets using plain washers. For this test setup, the annular gears were preloaded against each other by up to $1,000 \mathrm{Nm}$ and located. The planet carriers were subjected to a drive speed of up to $6,000 \mathrm{rpm}$. This resulted in a maximum planet gear speed of 20,000 rpm.

The measurement results in Figure 10 show the mean and maximum values of the axial bearing and plain washer temperatures and the corresponding temperature differences. A comparison of the results for an input moment of $100 \mathrm{Nm}$ and at a speed of $6,000 \mathrm{rpm}$, and for an input moment of $1,000 \mathrm{Nm}$ and at a speed of 2,500 rpm shows significant differences between the two design variants. The mean temperature difference is $1.5^{\circ} \mathrm{C}$ in the first case and $3{ }^{\circ} \mathrm{C}$ in the second case. The maximum values even show a temperature difference of $4{ }^{\circ} \mathrm{C}$ and $5{ }^{\circ} \mathrm{C}$, respectively.

The reduced temperatures and frictional torques determined in the simulation of the entire transmission and in actual component tests of individual bearings (Figure 11) have a significant effect on the system's emission characteristics. For example, the calculated reduction in $\mathrm{CO}_{2}$ emissions of the entire transmission in the NEDC is around $1 \%$. Replacing the planet thrust washers in an automatic transmission comprising four planetary gear sets with axial bearings results in a reduction in $\mathrm{CO}_{2}$ emissions of $1 \%$ with additional costs amounting to less than ten euros. 


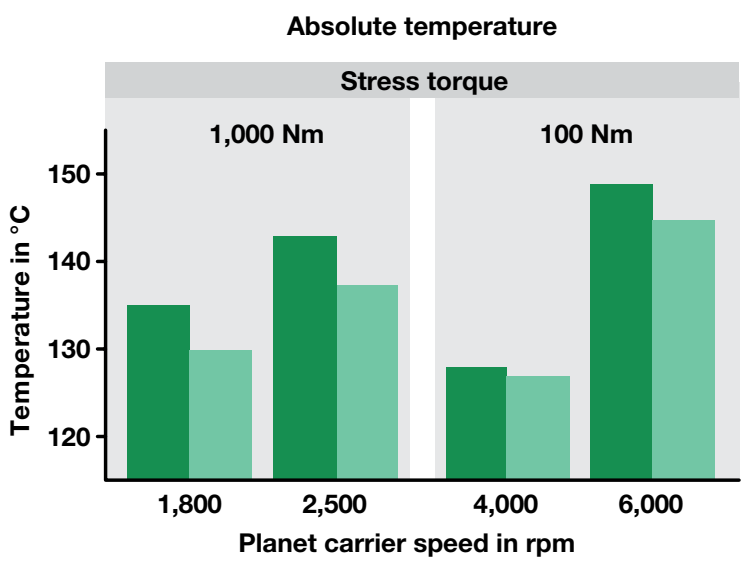

Thrust washer Axial needle roller bearing
Temperature difference

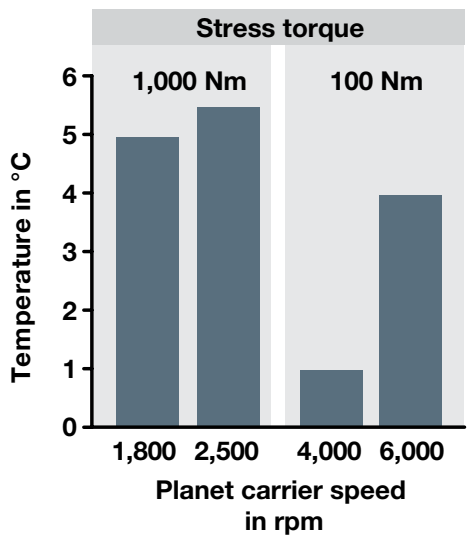

The planetary gear set system with the axial bearing was tested and compared with the plain washers on this test stand.

For this test setup, the annular gears were preloaded against each other by up to $1,000 \mathrm{Nm}$ and located (speed $0 \mathrm{rpm}$ ). The planet carriers reached a drive speed of up to $6,000 \mathrm{rpm}$.

The temperature is measured on the planet gear stud.

Figure $\mathbf{1 0}$ Functional tests on the planetary gear set test stand and results

\begin{tabular}{|l|c|c|c|c|c|}
\hline Investigations & Thrust washer & $\begin{array}{c}\text { Axial needle } \\
\text { roller bearing }\end{array}$ & $\begin{array}{c}\text { Reduction } \\
\text { in friction }\end{array}$ & $\begin{array}{c}\text { Reduction } \\
\text { in friction }\end{array}$ & $\begin{array}{c}\text { Reduction in } \\
\mathrm{CO}_{2} \text { emissions }\end{array}$ \\
\hline Simulation ( $3^{\text {rd }}$ gear) & 470 watts & 50 watts & 420 watts & $90 \%$ & $1 \%$ \\
\hline $\begin{array}{l}\text { Components test } \\
(6,000 \mathrm{rpm})\end{array}$ & $\begin{array}{c}0.23 \mathrm{Nm} \\
62\end{array}$ & $\begin{array}{c}0.02 \mathrm{Nm} \\
57^{\circ} \mathrm{C}\end{array}$ & $\begin{array}{c}0.21 \mathrm{Nm} \\
5^{\circ} \mathrm{C}\end{array}$ & $90 \%$ & $1 \%$ \\
\hline $\begin{array}{l}\text { Planetary gear set test } \\
(6,000 \mathrm{rpm})\end{array}$ & $150^{\circ} \mathrm{C}$ & $146^{\circ} \mathrm{C}$ & $4^{\circ} \mathrm{C}$ & - & - \\
\hline
\end{tabular}
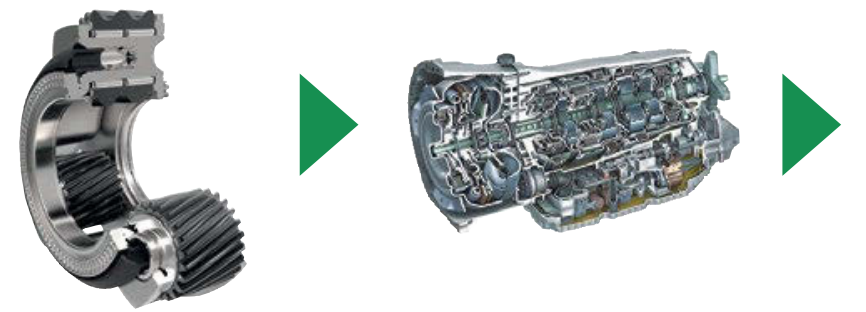

The axial needle roller bearings achieve a reduction in $\mathrm{CO}_{2}$ emissions of $1 \%$ with additional costs of only 10 euros

Figure 11 Effects on emission characteristics 


\section{Modular design in planetary gear sets}

Today, Schaeffler offers and supplies a large number of individual components and additional parts for planetary gear sets. Examples are the planet gear bearings that radially position the planet gear, support bearing forces and moments, and ensure rotation with minimal friction. The planet gear stud represents the inner raceway of the planet gear bearing. Schaeffler offers planet gear studs in all oil feed and geometry variants. The axial contact between the planet gear and the planet gear bearing is supported by the plain washers described above or by the newly developed axial needle roller bearings, which are also Schaeffler components. The plastic oil collector and the axial needle roller bearings running on the planetary gear set complement Schaeffler's product range (Figure 12).

The carrier usually comprises formed parts that are drawn and punched and have gear teeth manufactured by forming methods. Schaeffler uses its core exper- tise in these manufacturing technologies for a wide range of products, such as planet gear carriers or multi-disk clutch carriers.

Welding is the preferred joining method for manufacturing planet gear carriers. Schaeffler also has extensive experience in riveting technology, which is used for dual mass flywheels, torque converters or annulus carriers, for example. This expertise can also be used for the assembly of planet carriers. The advantages of riveting compared to thermal joining are that no thermal distortion occurs and no welding spatter must be removed due to welding. Schaeffler also develops the gears for planetary gear sets in-house in order to be able to offer comprehensive assemblies. During this development work, Schaeffler has gained a great deal of experience in high-performance planetary gear sets. Schaeffler has therefore been able to position itself as a development partner and supplier for mechanical assemblies or comprehensive solutions for planetary gear sets for manufacturers of entire transmissions.

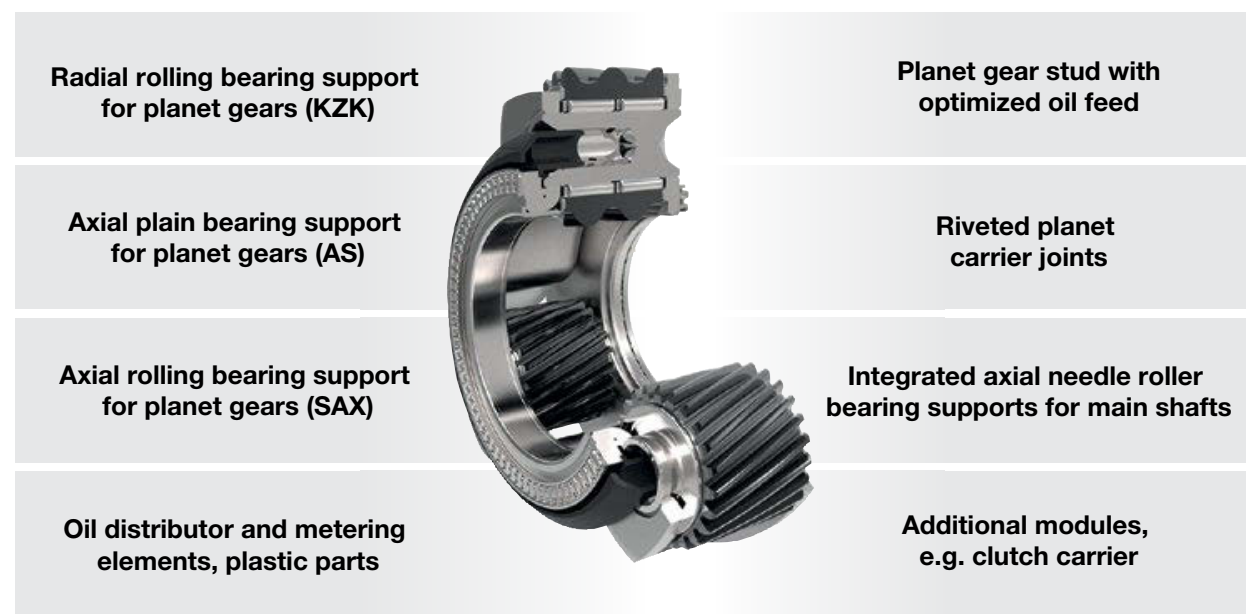

Figure 12 Schaeffler components with modular design for planetary gear sets 


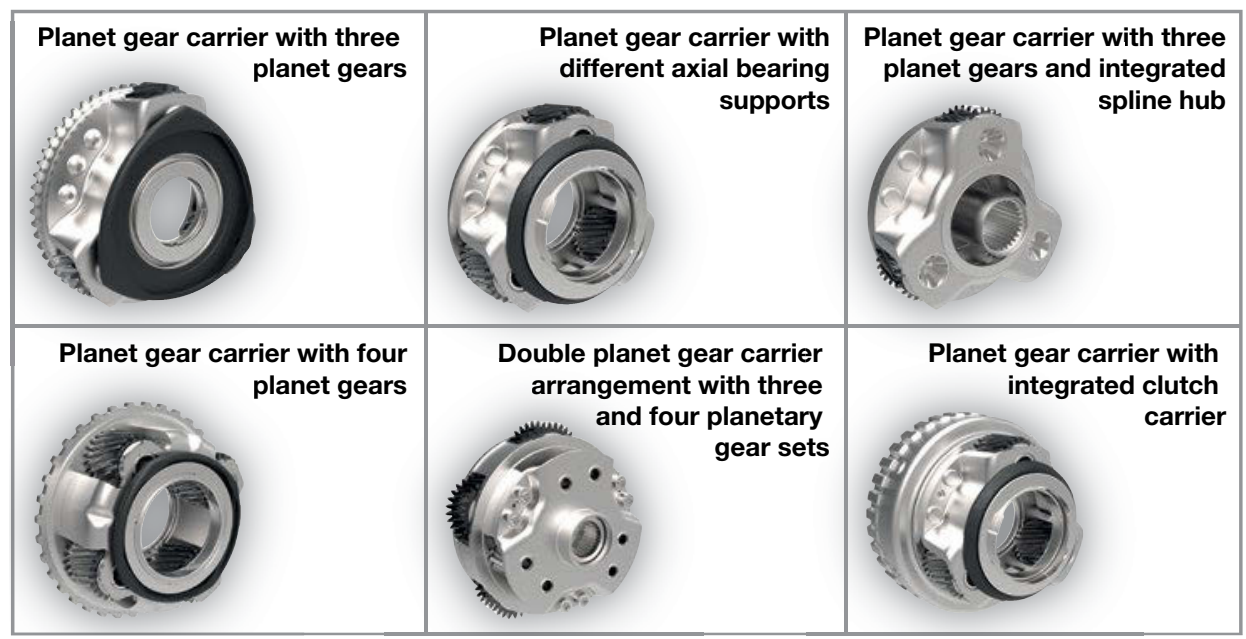

Figure 13 Variations in planetary gear set modules

Schaeffler offers modular concepts for planetary gear sets that can easily be integrated into existing transmission designs. The gear sets are characterized by gear teeth (spline teeth, engaging teeth) that are manufactured using forming methods. The planet carrier can be designed and manufactured using welding or riveting technology. Due to lower purchasing costs and shorter cycle times, riveting is particularly suitable if new machinery must be purchased. The entire gear set including additional parts is matched to the specific application in order to achieve the best possible oil lubrication and the lowest possible friction. The planet carriers can be designed with adjacent components such as multi-disk clutch carriers and annulus carriers, or integrated into load stages and differential stages (Figure 13).

Collaboration between Schaeffler's specialists and the transmission manufacturer early on in the concept phase is useful if the modular strategy is to develop its full potential.

\section{Literature}

[1] Scherer, H.; Wagner, G.; Naunheimer, H.; Dick, A.: Das automatische Getriebe 8HP70 von ZF. Getriebesystem, konstruktiver Aufbau und mechanische Bauteile. VDI reports 2029, Duesseldorf: VDI Verlag 2008

[2] Greiner, J.; Scherer, H.; Girres, G.; Dick, A.: Transmission Kit for Front-Wheel-Drive Applications from ZF. VDI reports 2130, Duesseldorf: VDI Verlag 2011

[3] Pabst, A.; Beeck, F.: Increasing the performance of planetary bearings for modern automatic transmissions. VDI reports 2158, Duesseldorf: VDI Verlag 2012

[4] Koch, O., Weber, J., Zintl, G., Gronau, B.; Energieeffiziente Auslegung von Wälzlagerungen, VDI conference on plain bearing and rolling bearing supports, 2011

[5] Koch, O., Gao, G.: Energy-efficient design of rolling bearings: An important contribution towards reducing $\mathrm{CO}_{2}$ emissions and increasing power density in automotive transmissions, CTI Symposium Innovative Automotive Transmissions and Hybrid \& Electric Drives, 2012

Open Access. This chapter is distributed under the terms of the Creative Commons Attribution Noncommercial License, which permits any noncommercial use, distribution, and reproduction in any medium, provided the original author(s) and source are credited. 